\title{
Lightweight Indoor Localization for 60-GHz Millimeter Wave Systems
}

\author{
Alain Olivier ${ }^{\S \sharp}$, Guillermo Bielsa ${ }^{\sharp \ddagger}$, Irene Tejado ${ }^{\sharp \ddagger}$, Michele Zorzi ${ }^{\S}$, Joerg Widmer ${ }^{\sharp}$, Paolo Casari ${ }^{\sharp \star}$ \\ $\sharp$ IMDEA Networks Institute, Madrid, Spain \\ ${ }^{\ddagger}$ Universidad Carlos III, Madrid, Spain \\ $\S$ Department of Information Engineering, University of Padova, Italy \\ ${ }^{\star}$ Corresponding author, email: paolo.casari@imdea.org
}

\begin{abstract}
In this paper, we target single-anchor localization schemes for millimeter wave (MMW) systems. The schemes are designed to be lightweight, so that even computationallyconstrained devices can support them. We identify the main propagation properties of MMW signals that have an impact on localization and design three algorithms that exploit these, namely a triangulation-validation procedure, an angle differenceof-arrival approach, and a scheme based on location fingerprinting. We evaluate the algorithms by means of simulations, and draw conclusions on their robustness. We then validate our results via measurements involving commercial pre-standard 60GHz MMW hardware. Our experiments confirm that, by relying only on a single anchor and without requiring complex signal processing at the receiver, the algorithms can localize a node with high probability, and in many cases with sub-meter accuracy. We conclude by discussing how these algorithms complement each other in terms of robustness and localization success probability.

Index Terms-Millimeter wave; localization; simulation; ray tracing; measurements; pre-standard hardware; $60 \mathrm{GHz}$
\end{abstract}

\section{INTRODUCTION}

Recent studies on the propagation characteristics of millimeter-wave (MMW) frequencies (in the 30 to $300 \mathrm{GHz}$ range) [1], [2], as well as advances in the design of RF circuits at these frequencies, have proven that MMW is a feasible communication technology, with the potential to relieve the impending crunch in the microwave spectrum and support new communication services for mobile users [3].

Moreover, the characteristics of radio propagation at MMW frequencies have interesting implications for the localization of radio terminals. Among the most significant, it has been shown that reflections do not lead to a substantial amount of scattering [4], and that the directivity of a transmitted beam is maintained after reflections with negligible scattering [5]. As a consequence, the equality of the angles of incidence and departure upon reflection, as predicted by Snell's law, holds in this case. Further investigations indicate that MMW propagation follows a quasi-optical pattern, in that the line-ofsight (LoS) component is predominant even in the presence of obstacles [6]. In addition, according to the Friis equation, the path loss can easily exhibit 30 to $40 \mathrm{~dB}$ more attenuation over typical link distances because of the short wavelength, and in some bands the channel presents further absorption peaks (e.g., at $60 \mathrm{GHz}$, due to oxygen absorption) [7]-[9]. Although such attenuation figures could be limiting for outdoor communications, they could turn out to be beneficial for indoor applications due to the possibility of confining the propagation of MMW signals inside a building or a room [10]. Finally, the higher path loss necessitates the use of narrow and very directional beams. These can be realized through high-gain horn antennas, as well as phased antenna arrays, which can be practically assembled within laptops or smartphones thanks to the order-of-millimeter wavelengths.

The MMW features briefly reviewed above require to rethink localization methods for indoor users employing MMW communication technologies, and to understand whether wellknown methods would be sufficient [11] or rather new schemes are required. Recent work [12] shows that indoor positioning continues to be a very active field of research. Precise location estimation indoors has a wealth of possible applications, such as augmented reality, tracking of patients in hospitals, personalized shopping, and so on. Moreover, precise knowledge of the terminals' positions could substantially improve the performance of physical- and MAC-layer communication protocols by supporting the management of the highly directional transmissions required in the MMW band [13].

In addition, to make localization a widespread service, accessible also for terminals with limited computational capabilities or strong energy constraints, the devised MMW localization algorithms must be lightweight and should not require a vast amount of signal processing to achieve sufficient accuracy. Indeed, the localization process can be aided in part by the anchor access point, which can relieve part of the environment estimation mechanism by broadcasting constraints such as room boundaries (walls, ceiling height, etc.) as well as its own position. However, even in this case, the localization algorithm should ideally have low computational complexity and should rely only on the information that can be directly passed on by a node's receiving hardware.

In this paper, we investigate localization schemes for MMW systems that specifically exploit the features of MMW propagation to provide accurate localization. We achieve this objective via three algorithms which offer different tradeoffs between precision and environment awareness requirements. All algorithms are designed to work with a single anchor node. This approach is promising in MMW scenarios, where the predominant power comes from the LoS component [6] unlike, e.g., in the case of ultra-wideband (UWB) signals in the 1-10 GHz band [14]. In addition, this relieves the need to deploy multiple anchors, and leverages instead on the richness of indoor multipath propagation [15] and on the capability to discriminate multipath components. Note that, even when the PHY layer cannot be modified to make AoA information avail- 
able, sector information for highly directional antenna arrays can be passed up to the MAC layer easily [13], making AoA practically available to higher-layer protocols. The feasibility and practicality of our schemes is demonstrated by means of simulations and validated via a number of experiments involving pre-standard commercial MMW systems operating in the $60 \mathrm{GHz}$ band.

This paper is organized as follows. The next section presents a review of indoor localization schemes. Section III describes the three proposed algorithms. Section IV discusses simulation results and draws initial conclusions on the performance of the algorithms. Section V presents an experimental campaign involving actual pre-standard MMW hardware, and presents the corresponding set of measurements to characterize the performance of our localization algorithms in real-world environments. Finally, we draw concluding remarks and propose future extensions in Section VI.

\section{RELATED WORK}

In this section, we survey related work on localization by subdividing the material into a general review of range-based and range-free schemes (Section II-A), a focus on recent advances in indoor localization (Section II-B), and a review of multipath-aided localization (Section II-C).

\section{A. Classic localization schemes}

Trilateration and multilateration are among the most common range-based location estimation techniques [11]. Trilateration requires to measure the distance of the node from a set of anchor points (a minimum of 4 to localize a node in the 3dimensional space) and to find the intersection of the spheres that result. The distance can be obtained by measuring the time of flight of signals, or otherwise estimated from the received signal strength (RSS) [16], provided that a precise path loss model is available. Multilateration relies on the knowledge of the Time Difference-of-Arrival (TDoA) at the node for a set of signals transmitted by different anchors. Computing the TDoA across different pairs of anchors identifies a set of hyperboloids, whose intersection yields the location estimate. Trilateration and multilateration heavily depend on the accuracy of the ranging process [17]: inaccurate ranging may hinder the identification of a unique intersection point, or even of any intersection at all.

Triangulation [11], in 2D, works by measuring the angles to the node from two anchors located at opposite ends of a baseline segment of known length. The location of the node is estimated as the third vertex of the resulting triangle. In $3 \mathrm{D}$, an additional anchor is required to compute the altitude of the node. Any errors on the estimation of the angles reflect negatively on accuracy, especially if the distance between the node and the anchors is large. Additionally, triangulation fails if the node is aligned with the anchors.

Localization based on location fingerprinting (FP) [11], [18] relies on the fact that radio waves emitted from one or more anchors yield a unique radio "fingerprint" at any given location: such fingerprint can be identified and employed to infer the location itself. While the definitions of fingerprint vary widely depending on the scenario and radio technology employed [11], the approach per se is often dismissed due to the heavy preliminary measurements required to construct a fingerprint database, and to the strong dependence of the localization error on the number of fingerprint measurements. Recent work has revived FP by suggesting that the database construction task can be crowdsourced [19].

The type of data available to the node to be located determines which method to apply. For example, trilateration would be applicable whenever the user can accurately estimate the distance between itself and a set of anchor nodes; conversely, triangulation may be preferable when the Angle-of-Arrival (AoA) of each multipath arrival from each anchor is known.

\section{B. Recent advances in indoor localization}

Recent work reconsidered WiFi-based indoor localization [20]-[22] via triangulation and multilateration-based schemes which assume a high density of Access Points (APs) and does not require to carry out any preliminary fingerprint measurement. In [23] the authors develop a localization system exploiting only one AP, where part of the complexity of the scheme is delegated to the user to be localized. The user is assumed to know his own orientation in space and the distance covered while moving by means of a digital gyroscope and accelerometer. In [24], a method is developed for the estimation of the TDoA based on the interference between Orthogonal Frequency-Division Multiplexing (OFDM) symbols from two different transmitters. In [25], the authors evaluate performance of RSS-, TDoA- and AoA-based localization schemes with MMW signals, assuming the presence of several anchor nodes deployed over a circumference around the receiver. It is observed that the AoA approach achieves the smallest localization error because of the broad AoA spectrum diversity originating from the circular geometry. In [26], an approach is proposed to harness the multipath propagation of WiFi signals for the purpose of locating a signal source, and simultaneously mapping the surrounding environment (to some extent). The approach is demonstrated using a USRP implementation of the algorithm and the related antenna array signal processing. The shorter wavelength of MMW with respect to microwave frequencies in the 1-10 GHz range makes it possible to pack a large number of antennas in a relatively small space. This means that both standard and massive MIMO approaches become feasible for localization [27].

\section{Multipath-aided localization in UWB channels}

UWB communications [28] have also been investigated in the scope of localizing static and mobile nodes, due to the possibility to separate multipath components in the channel impulse response. Building on the observation that deterministic multipath components typically constitute up to $90 \%$ of the UWB channel impulse response energy [14], the authors in [29] employ a single anchor node, and extract Time-of-Arrival (ToA) information from the AoA spectrum and estimate the distance from the anchor and the reflection points via a complex maximum likelihood approach. The case of multiple anchors is also considered in [30]. The 
knowledge of the floor plan is assumed in order to identify the location of VAs. In [31], Simultaneous Localization and Mapping (SLAM)-based approaches, which aim at relieving this assumption, achieved a median error below $5 \mathrm{~m}$.

Unlike most previous work, we target a MMW scenario and design practical localization algorithms that can be applied to signals transmitted by pre-standard $60-\mathrm{GHz}$ hardware. Our algorithms work under different assumptions, including the availability of floor plan information or lack thereof, and the presence of precise or imprecise AoA estimates, and are by design less complex than ML approaches.

\section{Millimeter Wave Localization Schemes}

In this section, we describe our three MMW localization algorithms. Our indoor localization scenario consists of a rectangular room bounded by four walls, the floor and the ceiling. We set up a three-dimensional Cartesian coordinate system centered in one of the corners of the room, and described by the canonical vectors of the three axes $\mathbf{e}_{x}=(1,0,0)$, $\mathbf{e}_{y}=(0,1,0)$ (oriented orthogonally along the floor sides), and $\mathbf{e}_{z}=(0,0,1)$ (oriented along the height of the room), such that any point $\mathbf{q}=q_{x} \mathbf{e}_{x}+q_{y} \mathbf{e}_{y}+q_{z} \mathbf{e}_{z}$ can be mapped to a triple $\left(q_{x}, q_{y}, q_{z}\right)$. The room boundaries and any other obstacles containing radio-reflective surfaces are grouped in the reflective objects set $\mathcal{Z}$. Obstacles are represented as threedimensional polyhedra with flat polygonal faces, straight edges and sharp vertices. We treat each face as an oriented surface $S$, represented by its normal vector $\mathbf{n}$, which can be found as

$$
\mathbf{n}=\frac{\left(\mathbf{p}_{2}-\mathbf{p}_{1}\right) \times\left(\mathbf{p}_{3}-\mathbf{p}_{1}\right)}{\left\|\left(\mathbf{p}_{2}-\mathbf{p}_{1}\right) \times\left(\mathbf{p}_{3}-\mathbf{p}_{1}\right)\right\|},
$$

where $\mathbf{p}_{1}, \mathbf{p}_{2}$ and $\mathbf{p}_{3}$ are points of the surface, $\|\cdot\|$ denotes the Euclidean norm, and $\times$ the cross-product. We assume that a single MMW AP is installed in the room at the location $\mathbf{p}_{\mathrm{tx}}$ and is employed as an anchor node for localization purposes. We also assume that the AP broadcasts information about its position and the characteristics of the objects in $\mathcal{Z}$ to the node(s) to be localized. This is in line with our working assumption that localization must be attainable even by devices with limited computational power or hard energy constraints. The algorithms are designed to leverage on the reflections of the AP signal off indoor boundaries in order to compute location estimates. The input to all algorithms is the Angle-of-Arrival (AoA) spectrum $P_{\mathbf{p}}(\alpha)$, which records the distribution (over the azimuthal plane) of the amplitude of multipath components at a given location $\mathbf{p}$, as a function of the azimuth $\alpha$, assumed to be measured relative to a reference angle $\alpha_{0} . P_{\mathbf{p}}(\alpha)$ is processed to yield a compact representation of different multipath components (MPCs) at p. In particular, a peak on the reception pattern is identified with an MPC [15]. Note that an MPC can be either a LoS path or a path that incurred (possibly multiple) reflections off one or more surfaces of the elements of $\mathcal{Z}$. We collect the MPCs in a $2 \times N_{\mathbf{p}}$ matrix $\mathbf{M}^{(\mathbf{p})}$, where $N_{\mathbf{p}}$ is the number of detected MPCs in $P_{\mathbf{p}}(\alpha)$. The first row of $\mathbf{M}^{(\mathbf{p})}$ contains the amplitude of each MPC sorted in decreasing order; the second row contains the AoA of the MPC, relative to $\alpha_{0}$. In this way, each column $\mathbf{M}_{:, k}^{(\mathbf{p})}$ of $\mathbf{M}^{(\mathbf{p})}$ (where the semicolon notation :, $k$ denotes all elements of the corresponding dimension, and in this case it means all rows of column $k$ ) can be seen as a vector in polar coordinates, departing from $\mathbf{p}$, where $\mathbf{M}_{1, k}^{(\mathbf{p})}$ and $\mathbf{M}_{2, k}^{(\mathbf{p})}$ denote the amplitude and phase of the vector relative to $\alpha_{0}$, respectively. We also assume that the room geometry (i.e., $\mathbf{p}_{\mathrm{tx}}$ and $\mathcal{Z}$ ) are known to the user to be localized. All algorithms developed in this paper localize a node on the azimuthal plane, but can be easily extended to the $3 \mathrm{D}$ case.

\section{A. Virtual anchors}

Each MPC belonging to the AoA spectrum $P_{\mathbf{p}}(\alpha)$ perceived at location $\mathbf{p}$ can be modeled as emitted by a virtual anchor that would be the source of a LoS signal reaching $p$ along the same AoA of the MPC. The position of the virtual anchor can be determined by mirroring the position of the AP with respect to the surfaces the signal has reflected off. Call $\mathcal{A}=\left\{\mathbf{a}_{0}, \mathbf{a}_{1}, \ldots\right\}$ the set containing the positions of the possible virtual anchors, and call $\overline{\mathcal{A}}=\left\{A_{0}, A_{1}, A_{2}, \ldots\right\}$ a partition of this set. We let $A_{0}=\mathbf{p}_{\mathrm{tx}}$, whereas each set $A_{i}, i=1,2, \ldots$ contains the virtual anchors that have been mirrored $i$ times with respect to any surface of the objects in $\mathcal{Z}$. Note that $\mathcal{A}$ and $\overline{\mathcal{A}}$ have countably infinite cardinality, as there is no limit to the number of times the AP can be mirrored. However, in practice the MMW signal of the AP will fade quickly as it propagates and reflects off the surfaces: this is a substantially different aspect with respect to, e.g., UWB systems at lower frequencies. Therefore, it makes sense to truncate $\mathcal{A}$ by considering a maximum reflection order $\mu$ [15]. To this end, we define $\mathcal{A}_{\mu}=\bigcup_{i=0}^{\mu} A_{i}$. Fig. 1 shows two virtual anchors $\mathbf{a}_{i}$ and $\mathbf{a}_{j}$ corresponding to a first- and a second-order reflection, respectively, hence $\mathbf{a}_{i} \in A_{1}$ and $\mathbf{a}_{j} \in A_{2}$.

\section{B. The Triangulate-Validate (TV) algorithm}

With this algorithm, a node at an unknown position $\mathrm{p}$ estimates its position via a number of triangulation steps followed by a validation of the estimated locations. The algorithm assumes that the nodes have measured the AoA spectrum $P_{\mathbf{p}}(\alpha)$ and have derived the matrix $\mathbf{M}^{(\mathbf{p})}$. We recall that the knowledge of $\mathbf{p}_{\mathrm{tx}}, \mathcal{Z}$ and $\alpha_{0}$ is also assumed. If the association between the anchors in $\mathcal{A}$ and the MPCs in $\mathbf{M}^{(\mathbf{p})}$ were known, it would be possible to directly triangulate the position of $\mathbf{p}$. As this association is unknown a priori, we estimate it via the procedure explained in the following, which is designed to be less complex than a ML approach [29].

With reference to the pseudo-code in Algorithm 1, we start by considering virtual anchors up to a reflection order $\mu$ (line 2). While a high value of $\mu$ would yield a richer virtual anchor set $\mathcal{A}_{\mu}$, a low value is more meaningful for triangulation: in fact, reflections weaken the signal, and virtual anchors of higher order can be quite far from the receiver. In turn, this distance would translate into a large triangulation error in the presence even of only small errors in the AoA spectrum. In addition, a signal at MMW frequencies is rarely distinguishable from noise after more than two reflections, hence we set $\mu=2$ in the following. 

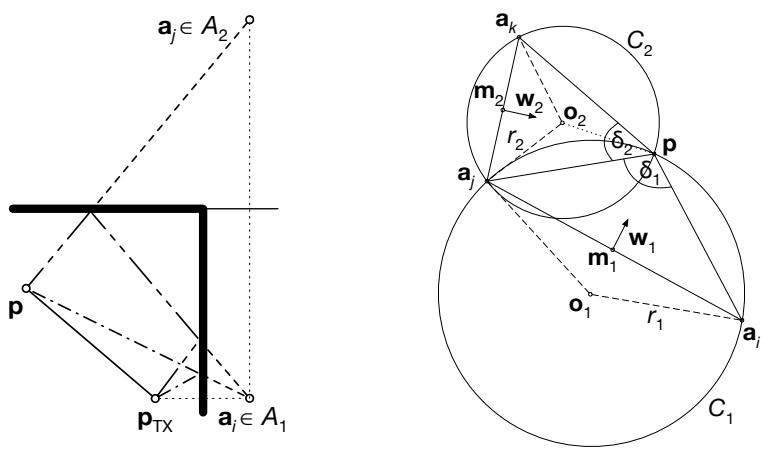

Figure 1. Virtual anchors related to Figure 2. The geometry of Angle first- and second-order reflections.

We start by considering $\mathbf{M}_{:, 1}^{(\mathbf{p})}$ and $\mathbf{M}_{:, 2}^{(\mathbf{p})}$ which, due to the sorting of $\mathbf{M}^{(\mathbf{p})}$, correspond to the MPCs with highest amplitude. Before using them to triangulate a position, we need to transform these entries into vectors departing from the position of any anchor, expressed relative to the reference Cartesian coordinate system of the room. This yields two vectors $\mathbf{u}_{1}=-\mathbf{Q} \mathbf{M}_{:, 1}^{(\mathbf{p})}$ and $\mathbf{u}_{2}=-\mathbf{Q} \mathbf{M}_{:, 2}^{(\mathbf{p})}$, where $\mathbf{Q}$ is the coordinate transformation (line 3). We now make an initial guess that the anchors from which $\mathbf{u}_{1}$ and $\mathbf{u}_{2}$ emanate are two points $\mathbf{a}_{i}, \mathbf{a}_{j} \in \mathcal{A}_{\mu}$ and triangulate a location over the azimuthal plane by solving the following linear system in two unknowns $t_{1} \geq 0$ and $t_{2} \geq 0$ (line 6):

$$
\mathbf{a}_{i}+\mathbf{u}_{1} t_{1}=\mathbf{a}_{j}+\mathbf{u}_{2} t_{2} .
$$

Call $\mathbf{p}_{k}, k \geq 0$ the position found. If $\mathbf{p}_{k}$ is valid with respect to some logical constraints (e.g., it is inside the room, not within any of the obstacles in $\mathcal{Z}$, and does not coincide with $\mathbf{p}_{\mathrm{tx}}$, line 7 ), we validate the position by basically measuring how compatible the remaining MPCs are with the positions of other virtual anchors in $\mathcal{A}_{\mu}$. We assign a weight $w_{k}>w_{\ell}$ to all anchors of partition subsets $A_{k}$ and $A_{\ell}$, $0 \leq \ell<k \leq \mu, A_{k}, A_{\ell} \subset \mathcal{A}_{\mu}$. This reflects the fact that the validation involving virtual anchors closer to $\mathbf{p}$ should be given greater importance. We now choose $\mathrm{v}_{\max }$ further MPCs $\mathbf{M}_{:, m}^{(\mathbf{p})}, 3 \leq m \leq \mathrm{v}_{\max }+2$, to be involved in the validation process. For each MPC, we consider all virtual anchors in $\mathcal{T}=\mathcal{A}_{\mu} \backslash\left\{\mathbf{a}_{i}, \mathbf{a}_{j}\right\}$ and associate a cost $c_{k}$ to $\mathbf{p}_{k}$ as follows:

$$
c_{k}=\sum_{m=3}^{v_{\max }+2} \min _{\mathbf{a} \in \mathcal{T}}\left[\cos ^{-1}\left(-\mathbf{u}_{m} \cdot \frac{\mathbf{a}-\mathbf{p}_{k}}{\left\|\mathbf{a}-\mathbf{p}_{k}\right\|}\right)\right]^{2} w_{\omega(\mathbf{a})},
$$

where $\mathbf{u}_{m}=-\mathbf{Q} \mathbf{M}_{:, m}^{(\mathbf{p})}$ and $\omega\left(\mathbf{a}_{n}\right)=\ell$ if $\mathbf{a}_{n} \in A_{\ell}$. We note that for a given $m$, the argument of the sum is identically zero if there exists an anchor $\mathbf{a} \in \mathcal{T}$ that lies exactly on the line leaving $\mathbf{p}_{k}$ with direction $\mathbf{M}_{:, m}^{(\mathbf{p})}$, whereas it increases if the minimum angle between any anchor $\mathbf{a} \in \mathcal{T}$ and $\mathbf{M}_{:, m}^{(\mathbf{p})}$, with corner in $\mathbf{p}_{k}$, increases. The square operation penalizes larger discrepancies more than smaller ones (lines 8 to 14). After computing the argument of the min in (3) the anchor a that minimizes the argument is removed from $\mathcal{T}$ (line 13).

The TV steps are repeated for all possible associations of $\mathbf{M}_{:, 1}^{(\mathbf{p})}$ and $\mathbf{M}_{:, 2}^{(\mathbf{p})}$ to the anchors in the set $\mathcal{A}_{\mu}$, returning a total of $K$ estimates $\mathbf{p}_{k}, k=1, \ldots, K$, and their related costs $c_{k}$

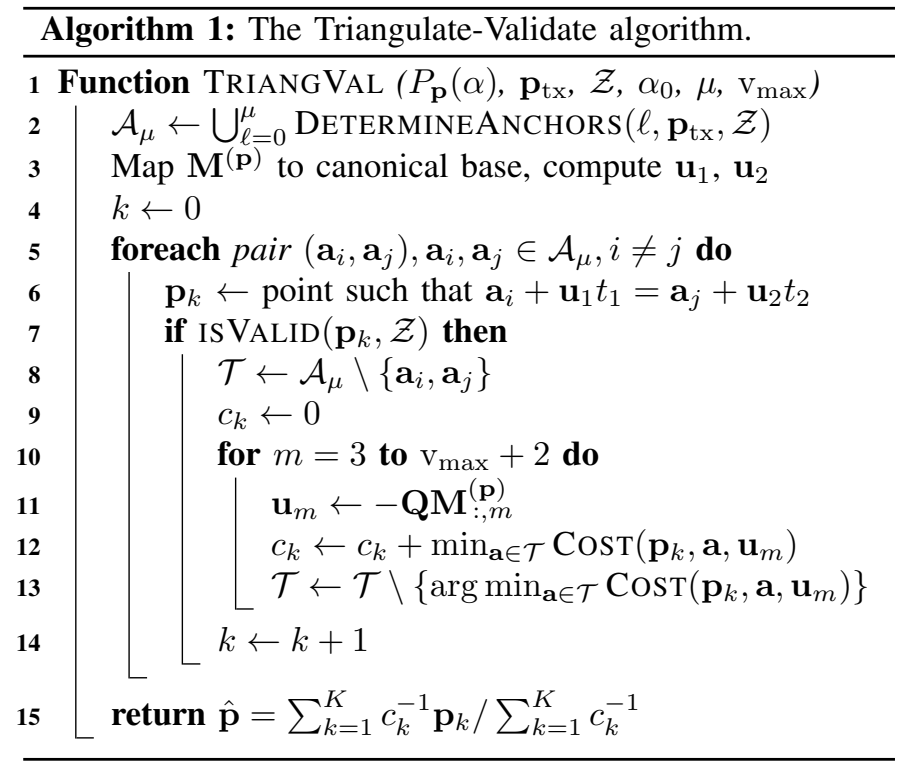

(line 5). Note that $K \leq\left|\mathcal{A}_{\mu}\right|\left(\left|\mathcal{A}_{\mu}\right|-1\right)$ since the algorithm fails if a triangulated position is found to be outside the room, or if the received AoA spectrum contains fewer than 3 MPCs. The final estimate of $\mathbf{p}$ returned by the TV algorithm is a weighed average of the positions $\mathbf{p}_{k}$ found along the process (line 15). In lines 12 and 13, the function $\operatorname{CosT}\left(\mathbf{p}_{k}, \mathbf{a}, \mathbf{u}_{m}\right)$, returns the argument of the min function in (3).

\section{The Angle Differences-of-Arrival (ADoA) algorithm}

The TV algorithm requires the knowledge of the reference angle $\alpha_{0}$, or equivalently, of the coordinate transformation matrix $\mathbf{Q}$ introduced in Section III-B. As this is not necessarily a feasible assumption, and the measurement of $\alpha_{0}$ (e.g., as provided by a smartphone's digital compass) may be affected by a significant error, we developed a second algorithm based on the Angle Differences-of-Arrival (ADoA) among MPCs. This algorithm is slightly more complex than TV, but is immune both to errors in $\alpha_{0}$ and to variations thereof across the room area.

We start by defining the angles $\delta_{1}=\mathbf{M}_{2,2}^{(\mathbf{p})}-\mathbf{M}_{2,1}^{(\mathbf{p})}$ and $\delta_{2}=\mathbf{M}_{2,3}^{(\mathbf{p})}-\mathbf{M}_{2,1}^{(\mathbf{p})}$. The ADoA algorithm is described in terms of the following geometrical problem, for which we refer to Fig. 2: given two points $\mathbf{a}_{i}$ and $\mathbf{a}_{j}$ in a 2D space, find the locus of the points $\mathbf{p}$ such that the angle $\widehat{\mathbf{a}_{i} \mathbf{p \mathbf { a } _ { j }}}$ (where $\mathbf{p}$ is the corner), is constant and equal to the angle $\delta_{1}$ defined above. This locus is a circumference, of which the segment $\overline{\mathbf{a}_{i} \mathbf{a}_{j}}$ is a chord. We assume the angle $\widehat{\mathbf{a}_{i} \mathbf{p \mathbf { a } _ { j }}}$ to be positive if $\mathbf{a}_{i}$ follows $\mathbf{a}_{j}$ in a counterclockwise direction within the space of a semi-circumference and $\left|\widehat{\mathbf{a}_{i} \mathbf{p \mathbf { a } _ { j }}}\right|<\pi$, where the $|\cdot|$ operator in this case represents the absolute value of the angle measure. With reference to Fig. 2, that depicts a typical ADoA localization scenario, consider the circumference $C_{1}$. Given the angle $\widehat{\mathbf{a}_{i} \mathbf{p \mathbf { a } _ { j }}}$, we have $\widehat{\mathbf{a}_{i} \mathbf{o}_{1} \mathbf{a}_{j}}=2 \widehat{\mathbf{a}_{i} \mathbf{p \mathbf { a } _ { j }}}$, where $\widehat{\mathbf{a}_{i} \mathbf{O}_{1} \mathbf{a}_{j}}$ is the central angle that insists on the same chord $\overline{\mathbf{a}_{i} \mathbf{a}_{j}}$. If $\widehat{\mathbf{a}_{i} \mathbf{p \mathbf { a } _ { j }}}>\pi / 2, \widehat{\mathbf{a}_{i} \mathbf{O}_{1} \mathbf{a}_{j}}$ is concave, hence $\left|\widehat{\mathbf{a}_{i} \mathbf{O}_{1} \mathbf{a}_{j}}\right|>\pi$. In this case, we wrap the angle back into the interval $[-\pi, \pi)$ via 


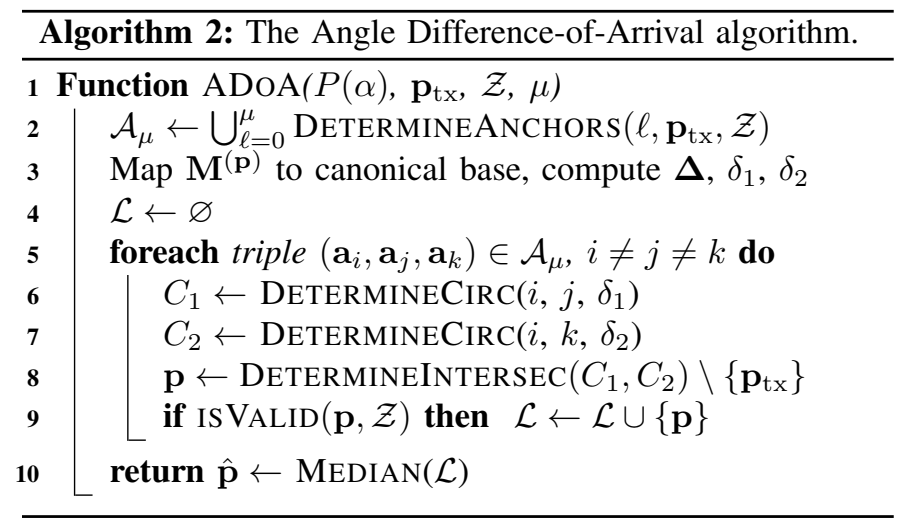

the operator $W(\cdot)$. The radius $r_{1}$ of $C_{1}$ can be found as

$$
r_{1}=\frac{\|\mathbf{a}-\mathbf{b}\|}{2 \sin \left(\left|W\left(\widehat{\mathbf{a}_{i} \mathbf{o}_{1} \mathbf{a}_{j}}\right)\right| / 2\right)} .
$$

Finally, given $\beta=r_{1} \cos \left(W\left(\widehat{\mathbf{a}_{i} \mathbf{o}_{1} \mathbf{a}_{j}}\right) / 2\right)$ and $\mathbf{u}=(\mathbf{a}-\mathbf{b}) \times$ $(0,0,1)$, the center of the circumference is found as

$$
\mathbf{o}_{1}=\mathbf{m}_{1}+\operatorname{sgn}\left(W\left(\widehat{\mathbf{a}_{i} \mathbf{o}_{1} \mathbf{a}_{j}}\right)\right) \frac{\beta \mathbf{u}}{\|\mathbf{u}\|},
$$

where sgn returns the sign of the angle, and $\mathbf{m}_{1}=\left(\mathbf{a}_{i}+\mathbf{a}_{j}\right) / 2$ is the middle point of the chord $\overline{\mathbf{a}_{i} \mathbf{a}_{j}}$. The vector

$$
\mathbf{w}_{1}=\left(\mathbf{o}_{1}-\mathbf{m}_{1}\right) \operatorname{sgn}\left(W\left(\left|\widehat{\mathbf{a}_{i} \mathbf{o}_{1} \mathbf{a}_{j}}\right|\right)\right)
$$

points to the section of $C_{1}$ defined by the chord $\overline{\mathbf{a}_{i} \mathbf{a}_{j}}$ where the constant angle requirement is satisfied. We now consider a second chord $\overline{\mathbf{a}_{j} \mathbf{a}_{k}}$ and the circumference $C_{2}$ as the locus of the points $\mathbf{q}$ where the angle $\widehat{\mathbf{a}_{j} \mathbf{q} \mathbf{a}_{k}}=\delta_{2}$. The radius $r_{2}$ and the center $\mathbf{o}_{2}$ of $C_{2}$ can be computed in the same way as above. The intersection $\mathrm{p}$ between $C_{1}$ and $C_{2}$ is considered a feasible location estimate whenever it is located on the sections of $C_{1}$ and $C_{2}$ pointed to by the orientation vectors $\mathbf{w}_{1}$ and $\mathbf{w}_{2}$. This means that, given the two chord centers $\mathbf{m}_{1}$ and $\mathbf{m}_{2}$, it must hold that $\mathbf{w}_{1} \cdot\left(\mathbf{p}-\mathbf{m}_{1}\right)>0$ and $\mathbf{w}_{2} \cdot\left(\mathbf{p}-\mathbf{m}_{2}\right)>0$.

The pseudo-code of the procedure that provides an estimate $\hat{\mathbf{p}}$ of the location of a node based on ADoA is given in Algorithm 2. The algorithm starts by determining $\mathcal{A}_{\mu}, \mathbf{M}^{(\mathbf{p})}$, $\delta_{1}$ and $\delta_{2}$ (lines 2 and 3). As for the Triangulate-Validate algorithm, we are initially unable to map each detected MPC to its related anchor node. Therefore, the ADoA algorithm collects a set of eligible positions which are the result of the intersection between the circumferences determined by the angle differences $\delta_{1}$ and $\delta_{2}$ and the chords $\overline{\mathbf{a}_{i} \mathbf{a}_{j}}$ and $\overline{\mathbf{a}_{j} \mathbf{a}_{k}}$, $\mathbf{a}_{i}, \mathbf{a}_{j}, \mathbf{a}_{k} \in \mathcal{A}_{\mu}$. Lines 6 to 8 determine the center and radius of $C_{1}$ and $C_{2}$ via (4) and (5), and compute their intersection by checking that it is feasible based on the orientation vectors $\mathbf{w}_{1}$ and $\mathbf{w}_{2}$ (see (6)). The ADoA procedure is repeated for different triples $\left(\mathbf{a}_{i}, \mathbf{a}_{j}, \mathbf{a}_{k}\right) \in \mathcal{A}_{\mu}$ (line 5) to yield a number of possible location estimates. Those positions that are valid with respect to the same logical constraints introduced for the Triangulate-Validate algorithm are collected in set $\mathcal{L}$ (line 9). Because resorting to angle differences does not require to estimate the reference angle $\alpha_{0}$, it may happen that for some combinations of anchor nodes, and in the presence of large

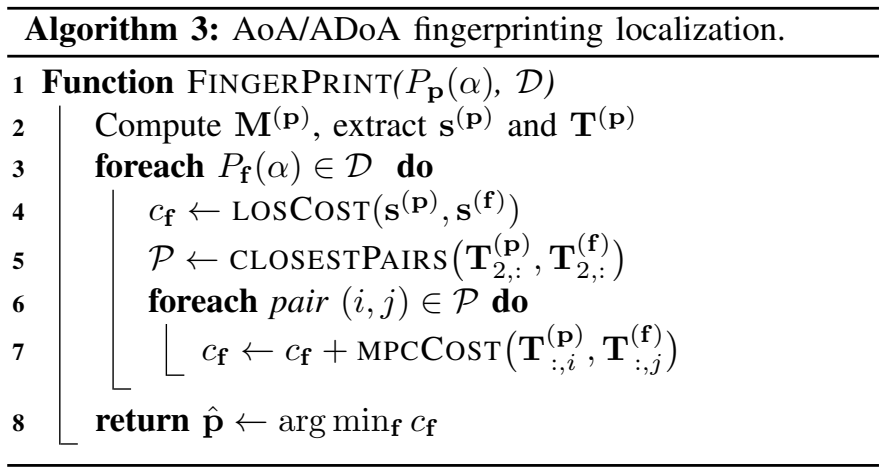

AoA estimation errors, the user is localized in an erroneous position that still satisfies the angle differences. To eliminate such spurious locations, the estimated position is computed as the median of $\mathcal{L}$ (line 8).

\section{Localization based on AoA/ADoA fingerprinting (FP)}

We finally present a fingerprint-based localization algorithm. Unlike TV and ADoA, FP does not strictly require that $\mathbf{p}_{\mathrm{tx}}$ and $\mathcal{Z}$ are known to the node to be localized. We assume that the area has been previously characterized by creating a database $\mathcal{D}$ of AoA spectra measured at a set of different locations $\mathcal{F}=\left\{\mathbf{f}_{1}, \mathbf{f}_{2}, \ldots\right\}$. Given a spectrum $P_{\mathbf{p}}(\alpha)$ measured at location $\mathbf{p}$ as an input, the algorithm looks up the most similar spectrum in $\mathcal{D}$ (according to some proximity measure) and returns its corresponding location. In order to be fair to the TV and ADoA schemes, we define the fingerprint of an AoA spectrum in terms of: $i$ ) a LoS feature, defined as the amplitude and AoA of the LoS arrival; and ii) a number of NLoS features, defined as the amplitude and ADoA (relative to the LoS AoA) of every other non-LoS (NLoS) MPC in the pattern. These choices are based on the fact that the strongest MPC is typically the LoS path [6], [15], and that we wish to make the feature matching process resilient to positiondependent errors in the reference angle $\alpha_{0}$. Given the pattern $P_{\mathbf{p}}(\alpha)$ and its MPC matrix $\mathbf{M}^{(\mathbf{p})}$, we identify the LoS feature as a vector $\mathbf{s}^{(\mathbf{p})}=\mathbf{M}_{:, 1}^{(\mathbf{p})}$. By calling $N_{\mathbf{p}}$ the number of columns in $\mathbf{M}^{(\mathbf{p})}$, we collect the remaining $N_{\mathbf{p}}-1$ entries of $\mathbf{M}^{(\mathbf{p})}$ in the NLoS feature matrix $\mathbf{T}^{(\mathbf{p})}$ and subtract the angle of the $\operatorname{LoS}$ path $\mathbf{M}_{2,1}^{(\mathbf{p})}$ from all elements of $\mathbf{T}_{2,:}^{(\mathbf{p})}$. In the pseudo-code in Algorithm 3, this corresponds to line 2.

Note that the AoA spectra received at any two different points typically have one LoS feature, and may have a different number of NLoS features. For each entry of $\mathcal{D}$ measured at location $\mathbf{f}$ (line 3 ), we execute an adapted instance of the closest point algorithm [32] which returns the indices $i$ and $j$ of the NLoS features in $\mathbf{T}^{(\mathbf{p})}$ and $\mathbf{T}^{(\mathbf{f})}$, respectively, whose angle differences are most similar. The similarity of the patterns at $\mathbf{p}$ and $\mathbf{f}$ is finally conveyed by the cost

$$
\begin{aligned}
c_{\mathbf{f}} & =-w_{\ell a}\left|\mathbf{s}_{2,1}^{(\mathbf{p})}-\mathbf{s}_{2,1}^{(\mathbf{f})}\right|-2 w_{p} \frac{\left|\mathbf{s}_{1,1}^{(\mathbf{p})}-\mathbf{s}_{1,1}^{(\mathbf{f})}\right|}{\mathbf{s}_{1,1}^{(\mathbf{p})}+\mathbf{s}_{1,1}^{(\mathbf{f})}} \\
& -\sum_{\forall(i, j)}\left(w_{n a}\left|\mathbf{T}_{2, i}^{(\mathbf{p})}-\mathbf{T}_{2, j}^{(\mathbf{f})}\right|+2 w_{p} \frac{\left|\mathbf{T}_{1, i}^{(\mathbf{p})}-\mathbf{T}_{1, j}^{(\mathbf{f})}\right|}{\mathbf{T}_{1, i}^{(\mathbf{p})}+\mathbf{T}_{1, j}^{(\mathbf{f})} \mid}\right),
\end{aligned}
$$


where the weights $w_{\ell a}, w_{n a}$ and $w_{p}$ are chosen to give more importance to the discrepancies in the angle differences of NLoS paths rather than to the absolute difference among the angles of the LoS path. This makes fingerprinting-based localization more robust to possible errors in the reference angle $\alpha_{0}$. On the contrary, the amplitude discrepancies of the paired LoS and NLoS features are given the same weight $w_{p}$. The function LOSCOST in line 4 computes the first line in (7), whereas the function MPCCOST repeatedly called in lines 6 and 7 computes the sum in (7). Finally, the algorithm returns the estimated position $\hat{\mathbf{p}}$ as the the location of the entry $\mathbf{f}$ in $\mathcal{F}$ that has minimum cost (line 8 ).

\section{PERFORMANCE EVAlUATION BY SimUlation}

In order to evaluate the performance of the localization algorithms in controlled ideal and non-ideal conditions, we have developed a ray tracer, which is an appropriate tool given the directionality of MMW transmissions [6], [15] and the limited amount of scattering generated by reflections [4]. The ray tracer simulates the propagation of MMW given the location of the transmitter, its transmit beam pattern, and the boundaries of the propagation area. Given a ray departing from the transmitter at a given position $\mathbf{p}_{\mathrm{tx}}$ with a direction $\mathbf{u}$, we first consider that the ray incurs standard distance-dependent path loss $P L(d)$ [33] as

$$
P L(d)=P L_{F S}\left(d_{0}\right)+10 \gamma \log _{10}\left(\frac{d}{d_{0}}\right),
$$

where $d$ is the distance from the transmitter, $P L_{F S}$ is the free space path loss at a reference distance $d_{0}$ and $\gamma$ is the path loss exponent (set to $\gamma=2$ [15] here). Each ray may bounce off the boundaries of the room as it propagates. These bounces are modeled using Snell's law as per [4]. Reflections have been set to cause a constant power loss at each bounce. The rays that lead to an actual received power contribution at the receiver (or eigenrays) are finally identified as those that propagate sufficiently close to the receiver. The output of the ray tracer is a list of the eigenrays, which is finally translated into the AoA spectrum at the receiver at a given position $\mathbf{p}$, $P_{\mathbf{p}}(\alpha)$, which is the input to all localization algorithms.

\section{A. Simulation scenario and results}

Our simulation scenario consists of a rectangular room of size $8.9 \times 6.3 \times 3 \mathrm{~m}$. The reference of the Cartesian coordinate system is placed on the south-western corner of the room, where the $x$ axis extends towards east and the $y$ axis towards north. The walls, floor and ceiling are modeled as uniform flat surfaces. We set $\alpha_{0}=\pi / 2$, i.e., angles are measured in a counterclockwise direction starting from the south-to-north direction. The transmitter is located at $\mathbf{p}_{\mathrm{tx}}=(0.2,0.2,1) \mathrm{m}$. We characterize the performance of the algorithms via the empirical cumulative distribution function (cdf) of the localization error, and in terms of the localization failure probability $P_{u}$. The cdf of the localization error is reported in Fig. 3 for the TV, ADoA and fingerprinting algorithms. For all of them, we truncate $\mathcal{A}$ to $\mathcal{A}_{\mu}$ by considering only the virtual anchor nodes that correspond to up to $\mu=2$ reflections.

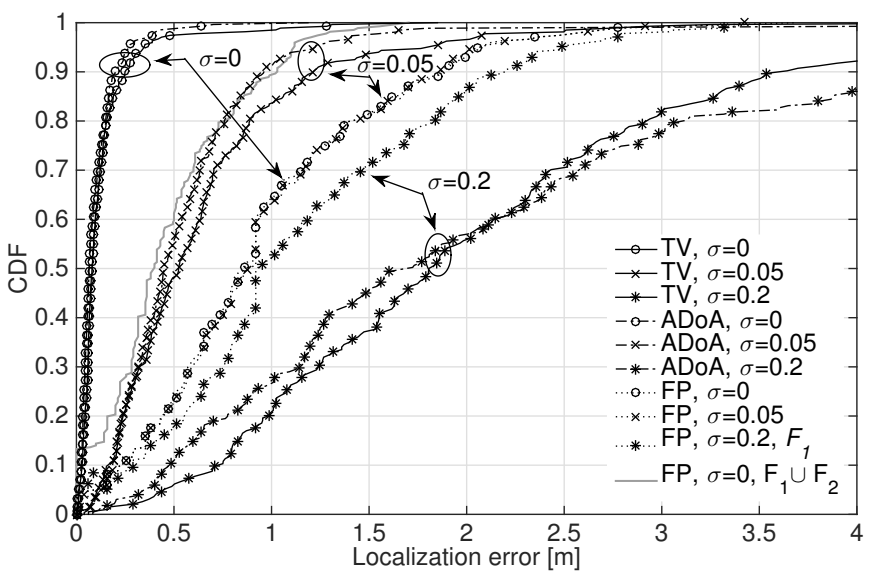

Figure 3. Localization error cdf for all algorithms for different values of the MPC AoA estimation error $\sigma$.

The fingerprinting database $\mathcal{D}$ is populated with simulated AoA spectra retrieved at the points in the training set $\mathcal{F}_{1}=$ $\{(1.85+1.3 k, 1.85+1.3 n, 1), k=0, \ldots, 4, n=0,1,2\}$. We simulate the fact that the measurement of the AoA spectrum by the node to be localized is not ideal, but rather may be affected by errors on the reference angle $\alpha_{0}$ and on the absolute estimates of the AoA of the MPCs, e.g., as a consequence of small changes in the propagation environment, or due to imperfect beamforming of the phased antenna array at the receiver. This non-ideal behavior is modeled for a given position p by first estimating the true AoA spectrum, by extracting the MPC matrix $\mathbf{M}^{(\mathbf{p})}$ and by adding a different random Gaussian-distributed displacement of standard deviation $\sigma$ to the elements of $\mathbf{M}_{2,:}^{(\mathbf{p})}$. The results in Fig. 3 are provided for $\sigma=0.05 \mathrm{rad} \approx 2.86^{\circ}$ and for $\sigma=0.2 \mathrm{rad} \approx 11.45^{\circ}$.

In the ideal case where $\sigma=0$, both the TV and the ADoA algorithms localize nodes with sub-meter accuracy, where the 90th percentile of the localization error is about $0.25 \mathrm{~m}$. In this respect, they consistently outperform FP, which experiences a double maximum localization error and sub-meter accuracy up to the 65th percentile. For increasing values of $\sigma$, the performance of all algorithms degrades. For $\sigma=0.05 \mathrm{rad}, \approx 90 \%$ of TV's and ADoA's estimates achieve sub-meter accuracy. For considerably larger AoA estimation errors, corresponding to $\sigma=0.2 \mathrm{rad}$, location estimates become expectedly less accurate, with a median error of $\approx 1.7 \mathrm{~m}$ for both ADoA and TV. Setting $\sigma=0.1 \mathrm{rad}$ would translate into a median localization error around $1 \mathrm{~m}$, and has been omitted for clarity in Fig. 3. For the TV algorithm, the error increases both because the triangulation step relies on sufficiently accurate AoA information, and because the estimated AoAs of the MPCs do not exactly correspond to the virtual anchors in $\mathcal{A}_{\mu}$. For the ADoA algorithm, instead, angle differences are affected by errors of variance $2 \sigma^{2}$, and negatively affect the estimation of the intersection between the circumferences in Fig. 2. Thanks to the matching operation carried out by the closest pair algorithm (see Algorithm 3), FP is more robust to high values of $\sigma$, and its performance does not degrade significantly even for $\sigma=0.2$. The localization error incurred 


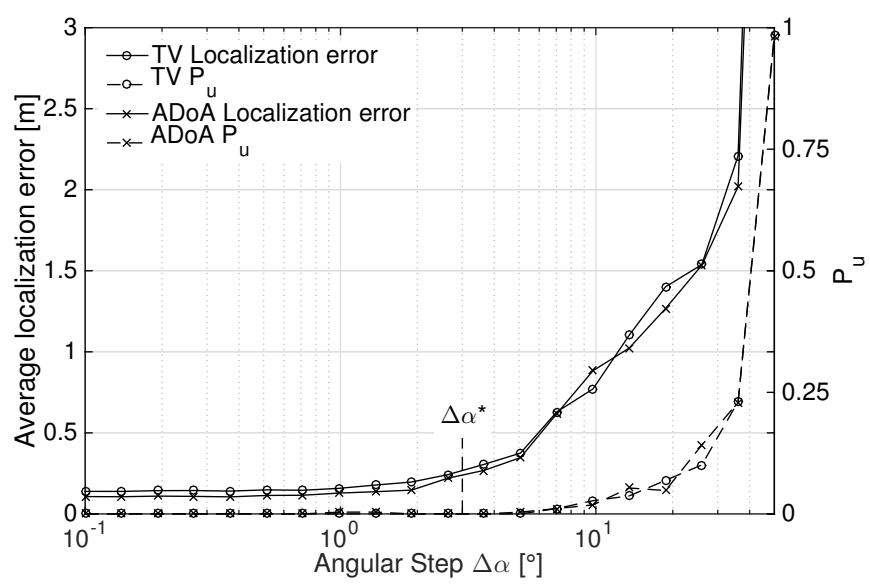

Figure 4. Localization error and probability of localization failure $P_{u}$ for the TV and the ADoA algorithms as a function of the angular step $\Delta \alpha$. The granularity chosen for the measurements in Section $\mathrm{V}$ is marked as $\Delta \alpha^{*}$.

by $\mathrm{TV}$ and $\mathrm{ADoA}$ is lower than that of fingerprinting for $\sigma=0$ and $\sigma=0.05 \mathrm{rad}$, and becomes worse for $\sigma=0.2 \mathrm{rad}$. The localization error of the fingerprinting algorithm can be improved by increasing the cardinality of the training set. Call $\mathcal{F}_{2}=\{(1.2+1.3 k, 1.2+1.3 n, 1), k=0, \ldots, 6, n=0, \ldots, 3\}$. By populating $\mathcal{D}$ with the patterns measured at the points of $\mathcal{F}_{1} \cup \mathcal{F}_{2}$, the error of the FP algorithm improves to match that of TV and ADoA for $\sigma=0.05 \mathrm{rad}$.

Fig. 4 presents the performance of the TV and ADOA algorithms when the AoA spectra are sampled with a limited precision $\Delta \alpha$, for $\sigma=0$. The results show that increasing $\Delta \alpha$ causes both the average localization error and the localizarion failure probability $P_{u}$ to increase. Both the TV and the ADoA algorithms are affected by the increase of $\Delta \alpha$. Interestingly, we observe that for $\Delta \alpha \leq 10^{\circ}$ the average localization error remains lower than $1 \mathrm{~m}$, and $P_{u}<0.05$.

\section{EXPERIMENTAL RESULTS}

We now present our experimental evaluation of the localization algorithms. We start by describing the measurement equipment and methodology, and continue by presenting the measurement campaign and the localization results.

\section{A. Equipment and experimental methodology}

In order to evaluate the performance of our localization algorithms in the presence of actual MMW transceivers, we carried out a number of indoor measurements in a $8.9 \times 6.3 \times 3 \mathrm{~m}$ room, i.e., the same size considered for the simulations in Section IV. This room was chosen so that the LoS MMW signal and its reflections could be comfortably heard at all locations in the room. In larger rooms, the signal of the AP would be excessively attenuated, impeding single-AP localization. However, it would be easy to extend of our methods to the case of multiple APs, which would address this issue. The room is unfurnished, but it does contain fixtures and appliances which create significant reflections off their surfaces, such as metal plumbing and heating radiators, wall-mounted rack cabinets, and floor-mounted cable leads. Our MMW transmitter is a Dell D5000 wireless docking station, placed at $(0.2,0.2,1) \mathrm{m}$;

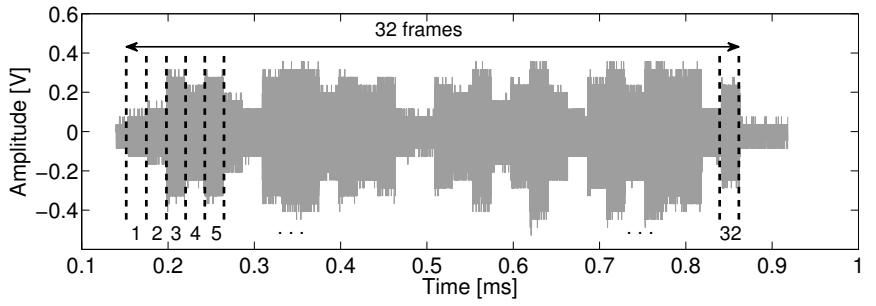

Figure 5. Dell D5000's device discovery frames. Each frame corresponds to a different configuration of the station's $2 \times 8$ phased antenna array [34].

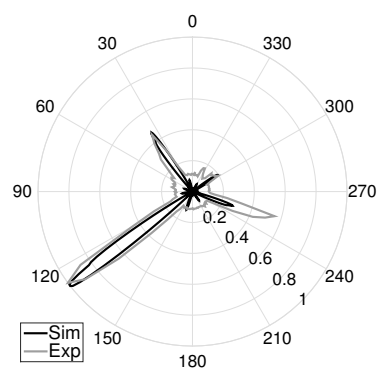

Figure 6. The AoA spectrum at $(5.75,4.45,1) \mathrm{m}$.

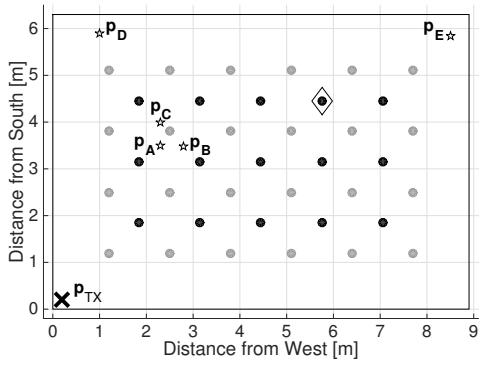

Figure 7. Experimental measurement grid. Black: $\mathcal{F}_{1}$; gray: $\mathcal{F}_{2}$; diamond: see Fig. 6. as a receiver, we employed the 60-GHz Vubiq development system, connected to an Agilent MSO-X 3034 oscilloscope and equipped with a standard $25 \mathrm{dBi}$-gain horn antenna mounted on an Arduino-controlled rotating stage. The D5000 periodically carries out a device discovery process according to the WiGig protocol. The process involves the transmission of 32 discovery frames, each corresponding to a different configuration of the station's $2 \times 8$ phased antenna array. An example of signal received during this process is shown in Fig. 5. These device discovery frames are sent every $102.4 \mathrm{~ms}$. More details are provided in [34].

The Vubiq system is employed to reveal LoS receptions as well as any MPC reflected off the boundaries of the room at any given position. This is done by redirecting the Vubiq's down-converted, $1.8 \mathrm{GHz}$-bandwidth, modulated signal traces of the analog I/Q output directly into the oscilloscope, which stores traces for later analysis. The process makes it possible to collect the amplitude of each of the 32 discovery frames over the azimuthal plane, for a number of orientations spaced by $\Delta \alpha^{*}=3^{\circ}$. This value has been chosen in order to achieve a reasonable tradeoff between measurement time and accuracy, and because Fig. 4 suggests that neither the localization error nor $P_{u}$ would be significantly affected. For every orientation, the receiver always captures the same number of frames as in Fig. 5, with different amplitudes that depend on the antenna patterns used by the D5000 for each frame, and on how the MMW signal is reflected off the boundaries and obstacles of the room. From these traces, an AoA spectrum is extracted by measuring the amplitude of the strongest frame. This yields robustness against the presence of small sidelobes in the transmitter's pattern. Fig. 6 shows one of the experimental patterns (in gray), measured at position $(5.75,4.45,1) \mathrm{m}$ (the point marked by a diamond in Fig. 7). We observe that the LoS path between the D5000 and the Vubiq has an AoA of $125^{\circ}$; 
Table I

$P_{u}$ AND PROBABILITY OF SUB-METER ACCURACY FOR ALL ALGORITHMS

\begin{tabular}{r|ccccc} 
& $\begin{array}{c}\text { TV } \\
\mu=1\end{array}$ & $\begin{array}{c}\text { TV } \\
\mu=2\end{array}$ & $\begin{array}{c}\text { ADoA } \\
\mu=1\end{array}$ & $\begin{array}{c}\text { ADoA } \\
\mu=2\end{array}$ & FP \\
\hline$P_{u}$ & 0.16 & 0.11 & 0.32 & 0.09 & 0 \\
$\mathbb{P}[$ error $\leq 1 \mathrm{~m}]$ & 0.76 & 0.70 & 0.82 & 0.72 & 0.57
\end{tabular}

strong reflections are detected with AoAs of $35^{\circ}$ and $250^{\circ}$, and a weaker reflection has an AoA of $300^{\circ}$. For comparison, we also plot an AoA spectrum simulated via ray tracing (in black), which proves the very good agreement of simulations and measurements.

\section{B. Measurement scenario and experimental results}

We carried out AoA spectrum measurements at the 39 grid points shown in Fig. 7, and at the 5 extra locations marked with a star. In the figure, the black and gray points respectively correspond to the sets $\mathcal{F}_{1}$ and $\mathcal{F}_{2}$ discussed in Section IV. The room was free from in-band interference during the whole realization of the measurement campaign. We tested the capability of the TV and ADoA algorithms to correctly localize a node located at each of the points in Fig. 7. For the fingerprinting scheme, we used the measurements at the points in $\mathcal{F}_{1}$ as a training set, whereas those taken at the points in set $\mathcal{F}_{2}$ were employed to test the algorithm. As in Section IV, we characterized the performance of the algorithms in terms of the cdf of the localization error and of the probability $P_{u}$ that a user cannot be localized. The TV and ADoA algorithms were configured to consider virtual anchors corresponding to up to either $\mu=1$ or $\mu=2$ reflections.

Fig. 8 shows the cdf of the localization error for all schemes. The best performance is achieved by the ADoA algorithm with $\mu=1$, for which $\approx 85 \%$ of the measurements have sub-meter accuracy. However, as reported in Table I, $P_{u}=0.32$ in this case. By allowing ADoA to process second-order reflections $(\mu=2)$, only in four cases does the algorithm fail to localize $\left(P_{u}=0.09\right)$. Being able to localize in more points, even those that are not localizable with $\mu=1$, comes at the cost of larger estimation errors, as the cdf correspondingly shows sub-meter accuracy in $75 \%$ of the cases. The TV algorithm also shows lower errors in the $\mu=1$ case with respect to the $\mu=2$ case. This is because in the received power patterns, most of the time, the strongest peaks are relative to the LoS and the first order reflections. In any event, the probability of being able to localize a node is better for $\mu=2\left(P_{u}=0.11\right)$ than for $\mu=1\left(P_{u}=0.16\right)$. The fingerprinting algorithm shows a higher localization error, although it achieves $P_{u}=0$. This is partly due to the choice of the measurement points in $\mathcal{F}_{2}$, which were taken at a maximal distance from the training set points in $\mathcal{F}_{1}$ in order to stress the FP algorithm. However, from the cdf we infer that about $55 \%$ of the points are localized correctly at one of the nearest neighbors in the training set, whereas others tend to be localized farther due to the similarity of the measured AoA spectra.

We conclude our analysis by showing the localization error of TV and ADoA ( $\mu=2$ in both cases) for each measurement point, and by marking which locations cannot be identified

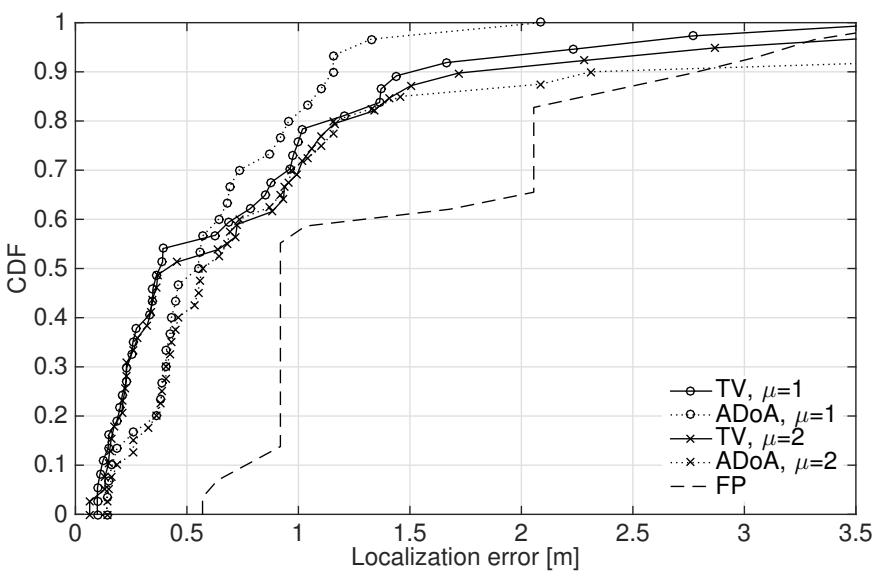

Figure 8. Experimental cdf of the localization error for all algorithms for different values of $\mu$.

by each algorithm. These features are rendered in Fig. 9 as a circle whose diameter is proportional to the error range. Those locations where the algorithms fail to estimate a position are indicated with a cross. Black markers refer to the TV algorithm, whereas gray markers refer to ADoA. Fingerprinting is not included in this graph due to the poor performance observed in Fig. 8. We observe that the largest localization errors are primarily due to the large widths of some arrivals in the measured AoA spectra, which make it difficult to accurately estimate the corresponding AoA. Specifically, for the locations near the transmitter, this is the case for the LoS AoA. Conversely, the weakness of the received signal and the presence of mainly second-order reflections in the AoA spectrum measured at the opposite corner of the room (point $\mathbf{p}_{E}$ ) explains why ADoA achieves a large error and TV is unable to localize. By comparing Fig. 8 and Fig. 3, we can also conclude that our measurements are affected by non-ideal conditions (related to the experimental equipment and setup) that induce an AoA estimation error with standard deviation on the order of $0.05 \mathrm{rad}$.

An interesting observation from Fig. 9 is that none of TV and $\mathrm{ADoA}$ is successful at all locations, but they complement each other in terms of successes and failures. The accuracy of these estimates depends mostly on AoA diversity, which in turn depends on the location of the failure. In addition, the experimental results in Fig. 8 suggest that it would be efficient to implement the algorithms so that they incrementally increase the cardinality of the anchor set $\mathcal{A}_{\mu}$ (and therefore their complexity) upon localization failures. In fact, for $\mu=1$, we found that there is a fair chance to be able to localize a node, and the resulting estimate would help achieve a very good accuracy; the less accurate but more robust version of the two schemes $(\mu=2)$ may be invoked only in order to deal with localization failures.

\section{CONCLUSIONS AND FUTURE WORK}

In this paper, we developed three different localization algorithms tailored around the characteristics of MMW propagation: one based on a Triangulate-Validate (TV) procedure, a second one based on Angle Differences-of-Arrival (ADoA), 


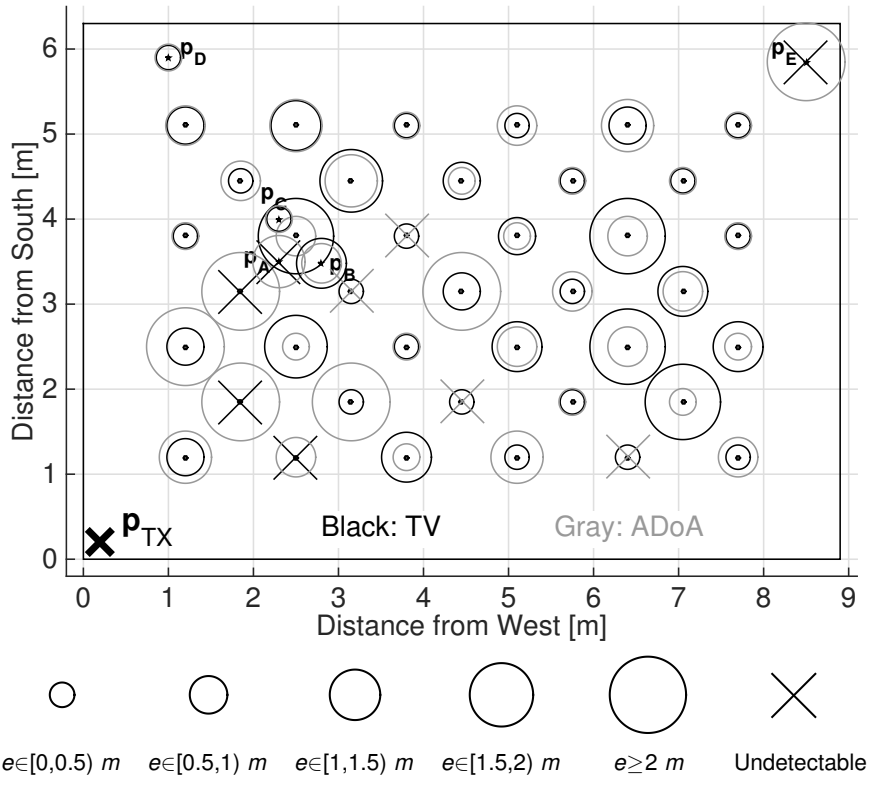

Figure 9. The measurements grid with the related errors in black for the Triangulate-Validate algorithm and in gray for the ADoA algorithm.

and a third one based on location fingerprinting (FP). We characterized the performance of the algorithms by simulations and by a set of measurements involving real MMW hardware operating in the $60 \mathrm{GHz}$ band. Our results show that unless a large error affects AoA estimations, our algorithms achieve sub-meter accuracy with high probability, and feature a very low probability of failing to localize. We observed that there is no absolute winner among the three algorithms, in that when one of them fails to localize, the others tend to succeed. While FP generally achieves a higher estimation error with respect to $\mathrm{TV}$ and $\mathrm{ADoA}$, these estimates tend to be robust against AoA estimation errors. Future work includes tests in a time-varying environment where propagation paths appear and disappear over time, and the use of a phased antenna array instead of a horn antenna at the receiver.

\section{ACKNOWLEDGMENTS}

This work has been supported in part by the ERC project SEARCHLIGHT, grant no. 617721, the Ramon y Cajal grant RYC-2012-10788, and the Madrid Regional Government through the TIGRE5-CM program (S2013/ICE-2919).

\section{REFERENCES}

[1] J. Thompson et al., "5G wireless communication systems: prospects and challenges," IEEE Commun. Mag., vol. 52, no. 2, pp. 62-64, Feb. 2014.

[2] S. Rangan, T. Rappaport, and E. Erkip, "Millimeter-wave cellular wireless networks: Potentials and challenges," Proceedings of the IEEE, vol. 102, no. 3, pp. 366-385, Mar. 2014.

[3] A. Osseiran et al., "Scenarios for $5 \mathrm{G}$ mobile and wireless communications: the vision of the METIS project," IEEE Commun. Mag., vol. 52, no. 5, pp. 26-35, May 2014.

[4] L. Subrt, P. Pechac, and S. Zvanovec, "New approach to modeling of diffuse reflection and scattering for millimeter-wave systems in indoor scenarios," PIERS Online, vol. 6, 2010.

[5] N. Peinecke, H. Doehler, and B. Korn, "Phong-like lighting for MMW radar simulation," in Proc. SPIE, Cardiff, UK, 2008.

[6] A. Maltsev et al., "Experimental investigations of $60 \mathrm{GHz}$ WLAN systems in office environment," IEEE J. Sel. Areas Commun., vol. 27, no. 8, pp. $1488-1499$, Oct. 2009.
[7] T. Rappaport et al., "Millimeter wave mobile communications for $5 \mathrm{G}$ cellular: It will work!" IEEE Access, vol. 1, pp. 335-349, 2013.

[8] G. R. MacCartney et al., "Path loss models for 5G millimeter wave propagation channels in urban microcells," in Proc. IEEE GLOBECOM, Atlanta, GA, Dec. 2013.

[9] T. Rappaport et al., "Broadband millimeter-wave propagation measurements and models using adaptive-beam antennas for outdoor urban cellular communications," IEEE Trans. Antennas Propag., vol. 61, no. 4, pp. 1850-1859, Apr. 2013.

[10] F. Giannetti, M. Luise, and R. Reggiannini, "Mobile and personal communications in the $60 \mathrm{GHz}$ band: A survey," Springer Wireless Personal Communications, vol. 10, no. 2, pp. 207-243, 1999.

[11] Hui Liu et al., "Survey of wireless indoor positioning techniques and systems," IEEE Trans. Syst., Man, Cybern. C, vol. 37, no. 6, pp. 10671080, Nov. 2007

[12] D. Lymberopoulos et al., "A realistic evaluation and comparison of indoor location technologies: Experiences and lessons learned," in Proc. IPSN, Seattle, WA, Apr. 2015.

[13] T. Nitsche et al., "Steering with eyes closed: mm-wave beam steering without in-band measurement," in Proc. IEEE INFOCOM, Hong Kong, China, Apr. 2015.

[14] P. Meissner et al., "Analysis of an indoor UWB channel for multipathaided localization," in Proc. IEEE ICUWB, Bologna, Italy, Sep. 2011.

[15] H. Xu, V. Kukshya, and T. Rappaport, "Spatial and temporal characteristics of 60-GHz indoor channels," IEEE J. Sel. Areas Commun., vol. 20, no. 3, pp. 620-630, Apr. 2002.

[16] B. Cook et al., "Indoor location using trilateration characteristics," in Proc. London Communications Symposium, London, UK, Sep. 2005.

[17] A. Zanella and A. Bardella, "RSS-based ranging by multichannel RSS averaging," IEEE Wireless Commun. Lett., vol. 3, no. 1, pp. 10-13, 2014.

[18] K. Kaemarungsi and P. Krishnamurthy, "Modeling of indoor positioning systems based on location fingerprinting," in Proc. IEEE INFOCOM, Hong Kong, China, Mar. 2004.

[19] C. Laoudias, D. Zeinalipour-Yazti, and C. G. Panayiotou, "Crowdsourced indoor localization for diverse devices through radiomap fusion," in Proc. IPIN, Montbeliard-Belfort, France, Oct. 2013.

[20] J. Xiong and K. Jamieson, "Arraytrack: A fine-grained indoor location system," in Proc. USENIX, Lombard, IL, Apr. 2013.

[21] Sen Souvik et al., "Avoiding multipath to revive inbuilding WiFi localization," in Proc. MobiSys, Taipei, Taiwan, Jun. 2013.

[22] R. Nandakumar, K. K. Chintalapudi, and V. Padmanabhan, "Centaur: Locating devices in an office environment," in Mobicom, Istanbul, Turkey, Aug. 2012.

[23] Alex Mariakakis et al., "SAIL: Single access point-based indoor localization," in Proc. MobiSys, Bretton Woods, NH, Jun. 2014.

[24] A. Jafari et al., "NLOS influence on $60 \mathrm{GHz}$ indoor localization based on a new TDOA extraction approach," in Proc. EuMC, Nuremberg, Germany, Oct. 2013.

[25] H. El-Sayed, G. Athanasiou, and C. Fischione, "Evaluation of localization methods in millimeter-wave wireless systems," in Proc. IEEE CAMAD, Athens, Greece, Dec. 2014.

[26] C. Zhang, F. Li, J. Luo, and Y. He, "iLocScan: Harnessing multipath for simultaneous indoor source localization and space scanning," in Proc. ACM SenSys, Memphis, TN, Nov. 2014.

[27] H. Deng and A. Sayeed, "Mm-wave MIMO channel modeling and user localization using sparse beamspace signatures," in Proc. IEEE SPAWC, Toronto, Canada, Jun. 2014.

[28] J. Kunisch and J. Pamp, "An ultra-wideband space-variant multipath indoor radio channel model," in Proc. IEEE UWST, Reston, VA, 2003.

[29] P. Meissner, C. Steiner, and K. Witrisal, "UWB positioning with virtual anchors and floor plan information," in Proc. WPNC, Dresden, Germany, Mar. 2010.

[30] P. Meissner et al., "Accurate and robust indoor localization systems using ultra-wideband signals," in Proc. ENC, Vienna, Austria, Apr. 2012.

[31] C. Gentner and T. Jost, "Indoor positioning using time difference of arrival between multipath components," in Proc. IPIN, MontbeliardBelfort, France, Oct. 2013.

[32] T. H. Cormen et al., Introduction to Algorithms. MIT Press and McGraw-Hill, 2001

[33] N. Moraitis and P. Constantinou, "Indoor channel measurements and characterization at $60 \mathrm{GHz}$ for wireless local area network applications," IEEE Trans. Antennas Propag., vol. 52, no. 12, pp. 3180-3189, 2004.

[34] T. Nitsche et al., "Boon and bane of $60 \mathrm{GHz}$ networks: Practical insights into beamforming, interference, and frame level operation," in Proc. ACM CoNEXT, Heidelberg, Germany, Dec. 2015. 